\title{
UNIVERSITY OF MINNESOTA.
}

School of Physics, Institute of Technology

THEORETICAL AND HIGH ENERGY PHYSICS PROGRAMS

\section{PROGRESS REPORT}

September 1, 1972 through August 31, 1973

U. S. Atomic Energy Commission Contract No. AT(11-1)-1764

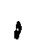

NOTICE

This report was prepared as an account of work sponsored by the United States Government. Neither the United States nor the United States Atomic Energy Commission, nor any of their employees, nor any of their contractors, subcontractors, or their employees, makes any warranty, express or implied, or assumes any legal liability or responsibility for the accuracy, com
pleteness or usefulness of ary information, apparatus, product or procēss disclosed, or represents that its use would not infringe privately owned rights.

1

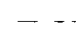




\section{DISCLAIMER}

This report was prepared as an account of work sponsored by an agency of the United States Government. Neither the United States Government nor any agency Thereof, nor any of their employees, makes any warranty, express or implied, or assumes any legal liability or responsibility for the accuracy, completeness, or usefulness of any information, apparatus, product, or process disclosed, or represents that its use would not infringe privately owned rights. Reference herein to any specific commercial product, process, or service by trade name, trademark, manufacturer, or otherwise does not necessarily constitute or imply its endorsement, recommendation, or favoring by the United States Government or any agency thereof. The views and opinions of authors expressed herein do not necessarily state or reflect those of the United States Government or any agency thereof. 


\section{DISCLAIMER}

Portions of this document may be illegible in electronic image products. Images are produced from the best available original document. 
This report describes research in nuclear physics and elementary particle physics. The nuclear research is all theoretical, but the high energy research is both theoretical and experimental. The report is organized according to this three-way division of our research activities.

The reader is warned that some of the results presented here are tentative and may be modified before publication. 


\section{NUCLEAR THEORY}

Finite-range, full-recoil calculation of the $\left({ }^{18} 0,{ }^{16} 0\right)$ reaction - B. Bayman

Experimental data on particle-transfer reactions induced by heavy projectiles have become available in recent years. At incident energies below the Coulomb barrier, the projectile follows a classical Rutherford orbit, and the nucleon transfer can be treated as a tunneling from the potential well of: one of the colliding fragments to the potential well of the other. At higher incident energies, the projectile and target get close enough for absorption effects to become important, and a more complete quantum-mechanical calculation is required. A natural approach is the generalization of the DWBA codes that were written for transfer reactions involving light projectiles $((d, p),(t, p)$, etc.).

Until the work described here, all heavy-fragment DWBA calculations relied on the no-recoil approximation. Let $r_{i}$ be the relative vector between the centers of mass of the target and incident projectile, and $r_{f}$ be the relative vector between the centers of mass of the fragments after the particie exchange has taken place. The no-recoil approximation sets $r_{\mathfrak{i}}=r_{f}$. This is a good approximation in the limit in which the mass transferred from one fragment to another is small compared to the masses of the fragments. In a realistic situation, e.g. the reaction ${ }^{62} \mathrm{Ni}\left({ }^{18}{ }_{0},{ }^{16} 0\right)^{64} \mathrm{Ni}$ with incident lab energy of $65 \mathrm{MeV}$ for the ${ }^{18} 0$, the difference between $r_{j}$ and $r_{f}$ in the important regions of configuration space is of the order of $0.5 \mathrm{~F}$. However, the wave lengths of the 180 and ${ }^{16} 0$ distorted waves are of the order of $1 \mathrm{~F}$, so $0.5 \mathrm{~F}$ errors in the magnitudes and orientations of $r_{\boldsymbol{j}}$ and $r_{\boldsymbol{f}}$ can have considerable effect on the DWBA matrix elements. 
A finite-range calculation of the $\left({ }^{18} 0,{ }^{16} 0\right)$ reaction has been performed, in which recoil effects are taken into account exactly. The 6-dimensional DWBA integrals were evaluated by the Monte-Carlo method. Seventy-five partial waves are included. The rather involved angular momentum couplings were handled using the techniques developed for the finite-range study of the $(t, p)$ reaction (B. F. Bayman and D. H. Feng, Nucl. Phys. A205 (1973) 513). Detailed comparison of the results of the calculation were made with the no-recoil calculation of Auerbach, Baltz and Kahana (Physical Review Letters 30 (1973) 1073). It was verified that the two calculations agree in the fictitions situation in which the mass of the transferred neutrons is negligitle compared to the fragment masses. However in the ${ }^{62_{\mathrm{Ni}}}\left({ }^{18}{ }_{0},{ }^{16} 0\right)^{64} \mathrm{Ni}$ situation, the no-recoil code was found to over-predict the differential cross-section by a factor of about 5. This information is important because the question of whether the $\left({ }^{18} 0,16_{0}\right)$ reaction is a one-step process depends upon the ability of this mechanism to account for the observed absolute cross-section, and hence we need reliable calculations of the absolute cross-section.

In the $(t, p)$ reaction, the two neutrons transferred from the triton are alms entirely in the singlet $(s=0)$ state. In the $\left({ }^{18} 0,{ }^{16} 0\right)$ reaction, the neutroris iransferred from ${ }^{18} 0$ have comparable singlet and triplet $(s=1)$ amplitudes. These contribute coherently to the reaction, and this coherence leads to some striking intensity rules. For example, the cross-section for transferring an $I=0$ pair from a $1 d_{3 / 2}$ orbit to a $2 p_{3 / 2}$ orbit is calculated to be about 50 times larger than if the transfer occurs from a $1 d_{3 / 2}$ orbit $i 0$ a $2 p_{1 / 2}$ orbit. In the case of the $(t, p)$ reaction, where the neutrons come from the $1 s_{1 / 2}$ orbit, the ratio of $\left(2 p_{3 / 2}\right)^{2}{ }_{I=0}$ to $\left(2 p_{1 / 2}\right)_{I=0}^{2}$ stripping is only 2 . 
Thus a comparison of $\left({ }^{18} 0,{ }^{16} 0\right)$ and $(t, p)$ transitions can yield spectroscopic information about the initial and final nuclear states.

A manuscript describing this work is now in preparation.

Odd-Even Absorption in $\mathrm{He}^{3}+\mathrm{He}^{3}$ Scattering and Level Structure of $\mathrm{Be}^{6},-\mathrm{Y}, \mathrm{C}$. Tang, D. R. Thompson (with J. A. Koepke and R. E. Brown)

Differential cross sections for $\mathrm{He}^{3}+\mathrm{He}^{3}$ elastic scattering are calculated at c.m. energies from 2.95 to $37.44 \mathrm{MeV}$ using the one-channel resonating-group method. A phenomenological imaginary potential, whose strength depends on whether the relative orbital angular momentum is even or odd, is included in order to account approximately for open reaction channels. The introduction of such an odd-even feature leads to a significantly improved agreement with experiment, especially at the larger scattering angles. In addition, an analys is of the calculated resonant phase shifts confirms recent experimental observations of highly excited levels in $\mathrm{Be}^{6}$.

Study of $d+{ }^{3} \mathrm{H}$ and $d+{ }^{3}$ He Systems with the Resonating-Group Method, - Y C. Tang, D. R. Thompson (with F. S. Chwieroth, R. E. Brown)

The one-channel resonating-group method, together wi th a phenomenological imaginary potential, is used to study the $d+3_{H}$ and $d+3_{\text {He systems. Our }}$ calculations show that, because of the Pauli exclusion principle, these systems possess the following rather interesting feature: in the $s=3 / 2$ state they behave similar to the $d+\alpha$ system, whereas in the $s=1 / 2$ state they behave more rike the ${ }^{3} \mathrm{He}+{ }^{4} \mathrm{He}$ system. Phase shifts are computed up to $\ell=7$, and an 
R-matrix analysis of these phases indicates that there are a large number of levels with resonance energies below $12 \mathrm{MeV}$. Differential scattering and totalreaction cross sections are compared with experimental data at several c.m. energies from about 2 to $14 \mathrm{MeV}$. This comparison shows that the introduction of an odd-even orbital-angular-momentum dependence into the imaginary potential can improve the agreement of the calculation with experiment.

Distortion Effects in $d+\alpha$ Scattering, - Y. C. Tang and D. R. Thompson

The deuteron specific distortion effect in the $d+\alpha$ problem is considered with a microscopic procedure. This procedure consists in the addition of square-integrable or distortion functions into the usual no-distortion resonatinggroup wave function. The result shows that with the consideration of this effect, a nucleon-nucleon potential which fits the low-energy two-nucleon scattering data and which yields a satisfactory agreement with the empirical $\alpha+\alpha$ phase shifts can be used to describe quite well the $d+\alpha$ experimental data. Also, it is shown that the specific distortion effect has an odd-even character; it has significant effects in even- $\ell$. states, but not in odd- $\ell$ states. Resonance structures are found in the partial-wave scattering amplitudes. These structures arise as a consequence of the coupling of the compound-nucleus states with the elastic-scattering channel.

Odd-Even Absorption and Coulomb-Exchange Effects in the ${ }^{3} \mathrm{He}+{ }^{4} \mathrm{He}$ and ${ }^{3} \mathrm{H}+{ }^{4} \mathrm{He}$ Systems - Y. C. Tang, D. R. Thompson (with J. A. Koepke, R. E. Brown)

Differential cross sections for ${ }^{3} \mathrm{He}+{ }^{4} \mathrm{He}$ elastic scattering are calculated at c.m. energies up to $44.5 \mathrm{MeV}$. using the one-channel resonating-group method. 
A phenomenological imaginary potential, whose strength depends on whether the relative orbital angular momentum is even or odd, is included in the calculation in order to account approximacely for open reaction channels. Exchange terms which arise from the nucleon-nucleon Coulomb interaction also are included. The introduction of both the odd-even absorption and the coulomb-exchange terms is found to lead to significantly improved agreement with experiment. In addition. the use of improved rms matter radii for ${ }^{3} \mathrm{He}$ and ${ }^{3} \mathrm{H}$ is found to yield ${ }^{2} \mathrm{P}$, boundstate energies for the ${ }^{3} \mathrm{He}+{ }^{4} \mathrm{He}$ and ${ }^{3} \mathrm{H}+{ }^{4} \mathrm{He}$ systems which are more consistent with experiment than found previously.

Effects of Imaginary Potentials on Spurious Resnnances in Resonating-group Calculations - Y. C. Tang, D. R. Thompson (with F. S. Chwieroth)

By studying the coupled-channel $d+{ }^{3} \mathrm{He}$ and $\mathrm{p}+\alpha$ resonating-group calculation, it is found that the introduction of phenomenological imaginary potentials is a simple way to eliminate spurious resonances from resonating-group and perhaps other mi croscopic reaction calculations.

Microscopic Coupled-Channel Study of the Five-Nucleon System - Y. C. Tang, D. R. Thompson (with F. S. Chwieroth)

A coupled-channel study of the five-nucleon system is performed with the resonating-group method. The channels which are explicitly considered are the $d+\mathrm{He}^{3}$ (or $\left.d+\mathrm{H}^{3}\right)$ and the $\mathrm{p}+\alpha($ or $n+\alpha)$ channels. Three- and morebody breakup channels are not included in the calculation, but their effects are crudely taken into account by the introduction of phenomenological imaginary potentials into the formulation. The nucleon-nucleon potential used is purely 
central, but does yield correct values for the two-nucleon effective-range parameters. Differential scattering and reaction cross sections are calculated at various energies. The result shows that for the $\mathrm{d}+\mathrm{He}^{3}$ and $\mathrm{p}+\alpha$ differential scattering cross sections, the calculated values agree in general quite well with the experimental data. For the $\mathrm{d}+\mathrm{He}^{3}+\mathrm{p}+\alpha$ differential reaction cross sections; the comparison between theory and experiment is somewhat less satisfactory. Although the general features of the experimental angular distributions are well reproduced, the magnitude of the calculated cross section is somewhat too low.

\section{PUBLICATIONS}

1. "Non-Local and Majorana-Exchange Terms in the Optical Model", Phys. Rev. C6, 2057 (1972)

2. "Phenomenological Study of S-Shell Hypernuclei with $\Lambda N$ and $\Lambda N N$ Potentials", Nucl. Phys. B47, 109 (1972).

3. "Odd-Even Absorption in ${ }^{3} \mathrm{He}+{ }^{3} \mathrm{He}$ Scattering and Level Structure of "Be", Nuc1. Phys: A201, 301 (1973).

4. "Study of $d+{ }^{3} \mathrm{H}$ and $\mathrm{d}+{ }^{3}$ He Systems with the Resonating-Group Hethod", Phys. Rev. C3, 938 (1973).

5. "Distortion Effects in $d+\alpha$ System", will appear in Nov. 1 issue of Phys. Rev. C.

6. "Effects of Imaginary Potentials on Spurious Resonances in Resonating-Group Calculations", will appear in Phys. Letters.

7. Microscopic Coupled-Channel Study of the Five-Nucleon System with the Resonating-Group Method", will appear in Phys. REv. C. 


\section{ELEMENTARY PARTICLE THEORY}

Studies in Quantum Field Theory - S. Gasiorowicz

The work that I did during the period covered by this progress report dealt primarily with quantum field theory. The utility of the path integral formalism in the quantization of non-abelian gauge theories led me to the examination of that formalism for other purposes. (a) I studied spontaneously broken symmetries in that formalism. Mly conclusions differ from those of Umezawa et.al: the Goldstone limit has nothing to do with the convergence factor needed to define the path integral. Convergence is achieved by the $-i \varepsilon$ term in the mass. The Goldstone limit can best be defined by taking a theory with an explicit form for chiral symmetry breaking, and then letting the symmetry breaking term go to zero. In that limit the pion mass will approach zero. I tried to formulate the path integral so that it would lead to finite Green's functions. I was not able to accomplish this. One reason is that the path integral formalism is just a functional Fourier transform transcription of the form of the generating function

$$
e^{i} \int d x \mathscr{L}_{I}\left(\frac{1}{i} \frac{\delta}{\delta J(x)}\right) e^{-\frac{1}{2} \int d x d y J(x)_{\Delta_{F}}(x-y) J(y)}
$$

and here it is clear that the explicit form does not exhibit the topology of the Feynman graphs, upon which the different degrees of divergence depend . Whether a line hooks across one or two vertices makes a difference in the degree of convergence of the resulting integral but the above form says nothing about that. There remains the remote possibility that working in an aribitrary number of dimensions these distinctions will not emerge till the final steps of the formulation, and this is being looked at. (c) I have tried to look at some 
approximation methods for the path integral. The only simple approximation is a stationary phase evaluation which is equivalent to the one-loop correction to the tree approximation, and even that proved to be intractable. I spend some time on the study of the classical equations of motion. In the Euclidean domain, with the four-dimensional angular momentum equal to zero (and only in that case) the equation

$$
\left(m^{2}-\partial_{i} \partial_{i}\right) \phi-g \phi^{n}=0
$$

reduces to $\left(x_{i} x_{i}=p^{2}\right)$

$$
\partial_{p}^{2} \phi-m^{2} \phi+g \phi^{n}=-\frac{3}{p} \partial_{p} \phi
$$

and the general properties of this can be studied in the phase-plane $\left(\phi, \partial_{p} \phi\right)$. The equation

$$
\frac{d H}{d p}=-\frac{3}{p}\left(\partial_{p} \phi\right)^{2} \leq 0
$$

where

$$
H=\left(\partial_{p} \phi\right)^{2}-m^{2} \phi^{2}+\frac{2 g}{n+1} \phi^{n+1}
$$

shows the general characteristics of the motion of the point $\left(\phi, \partial_{p}, \phi\right)$ as $p$ increases. This, however, is of no help in studying the tree approximation, which requires the solution of the nonlinear equation in the presence of an arbitrary source. (d) I also investigated what can be learned from the equations of motion as they reflect on equations obeyed by generators of the one-particle irreducible vertex functions. For a $\phi^{4}$ theory, the generator of the connected Green's functions $\|[\mathrm{J}]$ defined by 


$$
e^{i W[J]}=<T \cdot e^{i \int J(x) \phi(x) d x}>
$$

obeys the equation

$$
\left(\square+\mu_{0}^{2}\right) \frac{\delta W}{\delta J(x)}+\frac{\lambda_{0}}{6}\left(-\frac{\delta^{3} H}{\delta J(x)^{3}}-3 i \frac{\delta W}{\delta J(x)} \frac{\delta^{2} W}{\delta J(x)^{2}}+\left(\frac{\delta H}{\delta J(x)}\right)^{3}\right)=J(x)
$$

A corresponding equation can be derived for $\Gamma[A]$ where

$$
A(x)=\delta(H / \delta J(x)
$$

and

$$
\Gamma[A]=U[J]-\int d x J(x) A(x)
$$

The equation can be solved in the one-loop approximation for constant fields $A$ yields an expression identical to the one obtained by Coleman and Heinberg by a direct summation of graphs. It is unfortunately not tractable in higher loop approximations, and thus presents no advantage over more direct approaches. (e) It appears to be possible to derive the eikonal approximation using the path integral formalism. For massive electrodynamics the vector field enters into the integrand of the path integral in a gaussian form, and the "elimination of the oscillators" leads to a term that looks exactly like an eikonal term, in the remaining integration over the fermi fields. Such an integration can only be carried out for Yukawa-like theories with neglect of meson-meson interactions. The limitations of the path integral formalism are again apparent in the iability to see, without detailed calculations, that the eikonal is not good for scalar theories. None of these studies led to anything sufficiently new to warrant publication. 
I spent some time studying the formulation of finite equations of motion in quantum field theory using the Callan-Symanzik equations. This work is still in progress. It is my intention to survey the use of these equations in recent developments in field theory, possibly to write a review article. In this connection I also studied the parquet approximation for a simple $O(n)$ theory.

Physical Significance of the Dual Part of the Proton-Proton Elastic Scattering Amplitude - D. A. Geffen (in collaboration with D. D. Coon, University of Pittsburgh)

We have been investigating the physical significance of a recent dual model for $\mathrm{pp}$ elastic scattering (Coon; Sukhatme, and Tran Thanh Van) which gives a remarkably good fit to $d \sigma / d t$ for large $-t$ over an enormous range of energies and which yields a remaining diffractive part for $s \sim 50(\mathrm{GeV})^{2}$ which is remarkably similar in shape to the dominantly diffractive, $d$ o/dt obtained at the CERN ISR for $\dot{s} \sim 2000(\mathrm{GeV})^{2}$. This dual model involves two resonance trajectories which have the form $\alpha(t)=-c \quad \ln (b-a t)$ and where a branch occurs beyond $t=b / a>0$. One, $\alpha_{\omega}(t)$, is easily associated with the $\omega-f$ exchange degenerate trajectory and has $\alpha_{\omega}(0) \cong 0.4$ with known recurrences at the correct values of $t$. The second trajectory, $\alpha_{x}(t)$ has a smaller slope, with recurrences appearing at larger values of $t$ than $\alpha_{\omega}(t)$. The couplings of the $x$ resonances to protons are considerably weaker than that of the $\omega$ (or $\rho$ ) to protons yet its intercept is significantly higher, i.e., $\quad \alpha_{x}(0) \simeq 0.8$. Consequently, $\alpha_{x}(t)>\quad \alpha_{\omega}(t)$ for $t \leq 0$ so that, despite its weak coupling to the protons, it dominates the dual amplitudes for sufficiently large $-t$, i.e., $-t>-t_{s}$, where $-t_{s}$ decreases as $s$ increases. 
He have made the hypothesis, therefore, that $\alpha_{x}$ represents a trajectory for exotic meson exchanges. Consider the following arguments:

(i) The Coon-Sukhatme-Tran Thanh Van model (hereafter CST model) is a successful dual model for the $p p$ scattering amplitude.

(ii) If the CST model is extended to include SU(3), we know that without assuming the existence of resonances (and exchanges) in "exotic" channels, i.e., an SU(3) 27 plet, etc., the solution will be in conflict with experiment. Furthermore, if duality applies to $p p \rightarrow p p$ then why not $p p \rightarrow \Delta \Delta$ ? If so, there must be exotic trajectories which include, in the exact SU(3) limit, at least degenerate 1,8 , and 27 representations.

(iii) The branch point for $a_{x}, b_{x} / a_{x}$ is almost four times the branch point for $\alpha_{\omega}, b_{\omega} / a_{\omega}$. If these branch points correspond to quark pair thresholds, then the $\alpha_{x}$ behavior is consistent with a $q 9 \bar{q} \bar{q}$ threshhold.

(iv) The weakness of the exotic resonances coupling strength is consistent with their not being directly observed. Similarly, they would not enter into Regge fits to scattering amplitudes that have been heretofore confined to $|\mathrm{t}| \leq 2(\mathrm{GeV})^{2}$.

Our relatively large intercept for $\alpha_{x}$ is unexpected and offers the exciting prospect that exotic Regge exchanges may be observable at sufficiently high energies for such unambiguous reactions such as $\bar{p} \bar{p} \rightarrow \bar{\Sigma}_{\bar{\Sigma}}+$ despite the relative weakness of its coupling. Since exotics only get in the way of dual models involving only mesons, it has been suggested by Rosner that the meson decouples (or couples very weakly) from exotic trajectories. We have examined a variety of data for meson-nucleon reactions and find that the evidence is as yet inconclusive since better data for $d \sigma / d t$ for larger $-t$ and $s \geq 40(\mathrm{GeV})^{2}$ are required. A comparison of $d \sigma / d t$ for $\pi p \rightarrow \pi p$ and $p p \rightarrow p p$ at NAL energies 
in the region $1 \leqslant-t \leqslant 3$ should show a much sharper diffractive dip for $\pi p$ than for $p p$ but in the neighborhood of the pp dip, provided the exotic exchange is absent for pions or significantly less than that for protons:

We have also made a rough analysis of $p+p+p+N^{*}(1520,1680)$, whose shape for $d \sigma / d t$ at $s \leq 50(\mathrm{GeV})^{2}$ and large - $t$ show a remarkable similarity to that for pp elastic. Exotic exchanges seem to be more significant for the former reactions and the diffractive part less dominant, if present at all.

An analysis of I-spin crossing relations has been made in order to extend the model to $\mathrm{np}$ elastic scattering. It is found that additional non-symmetric dual amplitudes (i.e., linking $\alpha_{x}$ to $\alpha_{\omega}$ ) are required in order to satisfy the crossing relations. Additional efforts in this direction will be necessary.

Phenomenology of the Diffractive Component of Proton Proton Elastic Scattering D. A. Geffen (in collaboration with D. D. Coon)

The dual model (Coon-Sukhatme-Tran Thanh Van) for the non-diffractive part of $d \sigma / d t$ for $p p$ elastic scattering enables one to extract from the data the diffractive part of $d \sigma / d t$ over a wide range of energies, i.e., at present $25 \leq s \leq 50,1000<s<3000(\mathrm{GeV})^{2}$. The following properties are notable: (i) The slope in $t$ for $d a d t$ is greater in the forward cone, $-t \leq 0.1(\mathrm{GeV})^{2}$. (ii) There is a sharp dip at all these energies and, almost independent of $S$, at $-t_{\text {dip }} \approx 1.4(\mathrm{GeV})^{2}$. If there is any s dependence, $-t_{\text {dip }} \sim 1.35, s=2000$, and $-t_{\text {dip }} \sim 1.2, s=40(\mathrm{GeV})^{2}$.

(iii) A comparison of $d \sigma / d t$ at $s=4.0$ and $s=2800$, assuniting the forms, (a). $d \sigma / d t=A(t)\left(s / s_{0}\right)^{2 d-2}$ and, $(b), d \sigma / d t=A(t) B(s)\left(s / s_{0}\right)^{2 d(t)-2}$, for a range of 
values of $t\left(i . e .-t=.5, .8,2.48\right.$, and $\left.3.05(\mathrm{GeV})^{2}\right)$. In $(a)$ we ignore the region $-t<.5$ and hence the $S$ dependence of $d \sigma / d t$ at $t=0$, while in (b) we take it into account with a simple multiplicative factor $B(s)$. Nevertheless, in both cases, an $\alpha(t)$ of the form $\alpha(t)=1-c \sqrt{-t}$ with $c_{a} \approx 0.20(\mathrm{GeV})^{-1}$ and $c_{b} \approx 0.25(\mathrm{GeV})^{-1}$ fits the $S$ dependence of do/dt quite well.

We propose therefore, that (i), (ii), (iii) are all linked by the hypothesis that $\alpha(t)$, the "Pomeron" trajectory defined by the Regge assymptotic limit of the elastic scattering amplitude, is of the self-reproducing, "bootstrap" form

$$
\alpha(t)=1-c \sqrt{-t}
$$

or is only approximately self-reproducing with

$$
\alpha(t)=1-C \sqrt{4 m_{\pi}^{2}-t}
$$

Both forms, (1) and (2), yield an effective larger slope in $d \sigma / d t$ for $-t<0.1$. Trajectories with $\sqrt{t}$ behavior have the property that the rate of shrinkage of the cut contributions (multiple scattering) is the same as that of the pole (Born) term. This behavior contrasts with the case of linear trajectories which predict a much greater rate of shrinkage for the pole term and hence $d \sigma / d t$ in the forward peak. This is the major mechanism for producing a dip movement inward (i.e., $-t_{\text {dip }}$ decreases) as s increases. For $\alpha=1-c \sqrt{-t}$, this major dip moving mechanism is absent and we predict, for these ranges of energies, an almost stationary or slightly outward moving dip with increasing energy. 
We have also observed that the simple form, $d \sigma / d t \sim A_{0} e^{B t-2 C \sqrt{-t} \ell n s / s 0}$, with $s_{0} \sim 10$ to $20(\mathrm{GeV})^{2}$, fits the forward peak $\left(-\mathrm{t} \leqslant 1(\mathrm{GeV})^{2}\right)$ for $p p$ elastic scattering at ISR energies surprisingly we11. This offers the hope that the structure of the diffractive part of the scattering amplitude is relatively simple. So far, however, no completely satisfactory fit over the full range of $t$ has been found.

Higher Symmetries and Baryon Resonances - J. Rosner

This was published in Proceedings of the XVI International Conference on High Energy Physics, Batavia, Illinois, 1972, vol. 3, p. 149. A comparison was made of the predictions of " $\ell$-broken SU(6) $\Delta \pi$, and $\mathrm{No}$ decays of nonstrange baryon resonances. This comparison indicated good agreement for D-wave decays of the $70, L=1$ multiplet and F-wave decays of the $\underline{56}, L=2$ multiplet. S-wave decays of the $\underline{70}, L=1$ and $P$ - wave decays of the $56, L=2$ were consistent with the theory, but not as convincing because of scarcity of data. Some of the rules of " $\ell$-broken SU(6) 1 " were explained, including its connection with the ${ }^{3} P_{0}$ quark pair picture suggested earlier by licu, Carlitz-Kislinger, Colglazier, Petersen, and the author. 
Question of Direct Measurement of the Quark Charge - J. Rosner (in collaboration with Glennys Farrar) - Published in Phys. Rev. D7, May 1973

Feynman has suggested that the average charge of hadrons produced in a particular kinematic region in deep inelastic lepton scattering reflects the charge of the struck constituent. It was shown that such hadronic tests for the quark charge depend on specific properties of the final-state interaction even when the conditions suggested by Feynman for the validity of these tests are satisfied. Such tests were used to distinguish among various classes of production models.

Asymptotic Behavior of trajectory functions and size of classical orbits J. Rosner (in collaboration with Shan-Jin Chang) - To be published in Phys. Rev.

The strong-coupling limits of several ladder-graph models were explored. A correlation was found between the power of the coupling constant appearing in the leading Regge trajectory $\alpha(t=0)$ and the size of classical orbits described by the coordinate-space Bethe-Salpeter wave function. Specificaliy, (i) for the $\phi^{3}$ theory with exchanged mass $m \neq 0$, the orbit radius $r_{0}$ approaches a fixed value, and $a(t=0) \sim g^{\frac{1}{2}}$; (ii) for $\phi^{3}, m=0, r_{0}$ grows linearly with $g$ and $\alpha(t=0) \sim g ;(i i i)$ for $\phi^{4}, r_{0} \rightarrow 0$ and the leading singularity is a fixed cut in t. Expansions about classical orbits are possible in the first two cases, and lead in lowest order to a harmonic oscillator equation from which corrections to the classical result may be derived. 
Short-Distance behaviour of Quantum Electrodynamics and the Callan-Symanzik

Equation for the Photon Propagator - J. Rosner (in collaboration with

Eduardo de Rafael). To be published in Ann. Physics.

The short-distance behaviour of the photon propagator was discussed within the context of the corresponding Callan-Symanzik equation. The Callan-Symanzik function $\beta(\alpha)$ was calculated in perturbation theory up to sixth order. It was found that

$$
B(\alpha)=\frac{2}{3}\left(\frac{\alpha}{\pi}\right)+\frac{1}{2}\left(\frac{\alpha}{\pi}\right)^{2}-\frac{121}{144}\left(\frac{\alpha}{\pi}\right)^{3}+O\left[\left(\frac{\alpha}{\pi}\right)^{4}\right] .
$$

The simplicity of this result is to be contrasted with a corresponding perturbation theory calculation of the Gell-Hann, Low function $\psi(z)$, whose sixth order coefficient contains the transcendental (3) (the Riemann zeta function of argument three). A mechanism of cancellations in the calculation of $B(\alpha)$ was found, and its validity proven to all orders in perturbation theory.

Further Evidence for a $70, L=2$ Baryon Multiplet - J. Rosner (in collaboration with D. Faiman and J. Weyers)

The recently discovered $f$ wave dominance of the decay $F_{35}(1877) \rightarrow \Delta \pi$ was interpreted as requiring mixing between $\underline{56}, L=2$ and $\underline{70}, L=2$ baryon multiplets. This conclusion was drawn in the context of a broken version of SU(6) $)_{H}$. Further predictions included; a) the existence of a second prominent $\mathrm{F}_{15} \pi \mathrm{N}$ resonance around $2 \mathrm{GeV}$ in mass and, b) the more probable dominance of the $1.9 \mathrm{GeV}$ enhancement seen in $\pi^{+} \mathrm{p} \rightarrow \Sigma^{+}(1385) \mathrm{K}^{+}$by $\mathrm{P}_{33}$ and $\mathrm{P}_{31}$ rather than $F_{37}$ or $F_{35}$ resonances. 
Resonances in high energy colliding lepton beams - $J$. Rosner. To be published in Proceedings of the VIII Rencontre de Moriond, Meribel-les-Allues, France, 1973, edited by J. Tran Thanh Van; Orsay, 1973.

Suggestions were made for experiments in $\mathrm{e}^{+} \mathrm{e}^{-} \rightarrow$ hadrons to test higher symmetries for resonances. These included studies of the $B, A_{1}$, and higher negative-parity mesons, as well as possibilities for testing $S ! 1(6)_{W}$ in $e^{ \pm} e^{-} \rightarrow e^{ \pm} e^{-} f_{0}$.

Phases of resonant amplitudes: $\pi N \rightarrow \pi \Delta-J$. Rosner (in collaboration with D. Faiman). To be published in Phys. Letters.

It was found that a recent phase shift analysis in $\pi N \rightarrow \pi \Delta$ is compatible with "l-broken SU(6) W" only if the continuation of the analys is across an energy gap $\left(1540 \mathrm{MeV} \leq \mathrm{E}_{\mathrm{c} . \mathrm{m} .} \leq 1650 \mathrm{MeV}\right)$ has been performed improperly. In that case, however, one finds good agreement with experiment. Based on our prediction, the group performing the analys is (LBL-SLAC) found that another continuation across the gap was indeed possible, and is including the new solution in a forthcoming publication.

Current quarks, constituent quarks, and symmetries of resonance decays - J. Rosner (in collaboration with A. J. G. Hey, J. Weyers). To be published in Nuclear Physics B.

The transformation between "current" quarks and "constitutent" quarks recently suggested by Melosh was examined with respect to its predictions for pionic decays of resonances. It implies the use of $S U(6)_{W}$ for classifying 
particle states but not for describing decay processes. Instead, pion emission proceeds via $\Delta L_{z}=0, \pm 1$, where $L$ is the internal ("quark") orbital angular momentum. This decay symmetry may be called $S U(6)_{W}\left[\Delta L_{z}=0, \pm 1\right]$. It was proven equivalent for any decay $A \rightarrow B+\pi$ (where $A, B$ are arbitrary $q q$ or $q q q$ hadrons) to the ${ }^{3} P_{0}$ quark-pair creation model for such decays, as formulated by Micu, Carlitz-Kislinger, Colglazier, Petersen and Rosner. The roles of final orbital angular momenta $l$ and of $S U(3) \times$ SU(3) subgroups of SU(6) $W$ were also discussed, and some new predictions were made for decays of meson resonances below $1700 \mathrm{MeV}$.

Hadron Spectroscopy - J. Rosner. To be published in Proceedings of the 1973 Berkeley Meeting, Division of Particles and Fields, American Physical Society.

Recent experimental and theoretical developments in the field of resonant particles were reviewed.

Classification and Decay of resonant particles - J. Rosner. To be published in Physics Reports.

This is an extensive review dealing with recent aspects of hadron spectroscopy, particularly with respect to symmetries higher than SU(3). Sections deal with new data, the status of SU(3), empirical regularities such as Regge trajectories, the quark model, SU(6) $\mathrm{f}$ and related symmetries for decays, duality and the $0^{+}$mesons. Two concluding sections describe some future experiments that may be helpful at low and high energies in making further progress in the area of resonant particles. 
Conservation Laws and Vlard-Takahashi Identities - H. Suura (in collaboration with Bing-Lin Young, Iowa State University). To be published.

Local variations of field variables in the action integral are used to derive various conservation 1aws. Different choices of variations lead to the form of the canonical energy-momentum tensor, the angular momentum tensor, the symmetric energy-momentum tensor and the Improved Energy Momentum Tensor of Callan, Coleman and Jackiw. The same local transformations are used to change integration variables in the functional integral representation for n-point functions, and this leads to the Ward-Takahashi identities that correspond to the conservation laws. The formal nature of the path integral form of the Green's functions is reflected in the fact that the anomalies in the identities do not emerge in this treatment.

Logarithmic Trajectories and the Magnitude of the Quark-Gluon Coupling Constant - H. Suura (in collaboration with D. Coon, Iniversity of Pittsburgh).

A fit to high energy proton-proton scattering data over a wide range of momentum transfer indicates that the non-diffractive part of the elastic scattering is described by a leading trajectory function $\alpha(t)$ that levels off like $\log (-t)$ for large $(-t)$, according to coon, Sukhatme and Tran Thanh Van. It is pointed out that such a logarithmic trajectory is consistent with vectorgluon exchange between quarks and antiquarks. Furthermore the coefficient of $\log (-t)$ is uniquely determined by the quark gluon coupling constant. Using the C.S.T. fit to the data, the coupling constant is determined to be $g^{2} / 4 \pi=15$. 
Long-Range Potential Model of Hadrons, and Ritz Variational Method in

Field Theory - H. Suura

The long-range potential model of hadrons proposed last year was further studied. The objective was to see whether the model could be justified in the framework of quantum field theory. It was found that the Bethe-Salpeter approach (summing ladders) does not yield the model, since there exists an upper limit to be coupling constant, preventing an arbitrarily strong binding. A new approach has been developed. The Ritz variational principle of nonrelativistic quantum mechanics has been generalized into a (mass) ${ }^{2}$ variational principle, using generalized Green's functions. The path integral representation. for the Green's functions is a natural tool in this variational method. The general approach works for free fields, and is being applied to the quark-gluon model.

P. Zucker

During the past year, much attention was directed at the problem of weak production of nucleon resonances: $\nu+N \rightarrow \ell^{-}+\mathbb{N}^{*}$. Previously, a detailed dynamical model had been published for these processes. In this model a dispersion relation was written for each partial wave and unitarity was used for the imaginary part. An approximate solution to the resulting Omnes equation was found which was appropriate to resonance formation. It had the simple N/D form of a Born term divided by a denominator function which provides the resonance enhancement. By using the conserved vector current theory, the 
weak process is related to electroproduction, thereby determining the magnitude of the denominator function. Elementary coupling constants and the elastic form factors are inputs into the calculation, thus leaving no adjustable parameters. This theory agreed well with the CERN data ${ }^{2}$. Within the past year, new results have been reported from an Argonne experiment ${ }^{3}$. During this time close touch was maintained with the Argonne group as comparisons of this and other models were made with the new (and more extensive) data. The model still agrees well, but the wealth of new data provided the opportunity to further investigate the effects of the partially conserved axial current hypothesis (PCAC)

The PCAC hypothesis relates the matrix elements of the divergence of the axial current to those of the corresponding pion process. Thus for $\nu+p+\mu^{-}+p+\pi^{+}$, the reaction reported at Argonne, the contribution from the divergence of the axial current is related to $\pi^{+}+p \rightarrow \pi^{+}+p$, a well-known reaction. In the energy region of this experiment, both are dominated by the $\Delta$ (1236) resonance. Thus, it would seem a simple matter to test PCAC directly in this experiment. Unfortunately, the other components of the axial vector currents contribute importantly and overwhelm the contribution from the divergence of the axial current. Only in the very small kinematic region near the forward direction (momentum transfer from the leptons $k^{2} \leqslant .01-.02 \mathrm{GeV}^{2}$ ) is the PCAC amplitude important. Since neutrino events are rare, there are not nearly enough in this region to provide a measurement of the cross section.

It was realized that the implications of PCAC could be studied with the help of such detailed models as the one discussed above. Lacking adjustable parameters, the previous model ${ }^{1}$ had the divergence of the axial current proportional to a pion pole, but the residue disagreed with the PCAC value. 
In order to build a model entirely consistent with PCAC, it was necessary to. adopt a slightly different approach and choose a particular value for the poorly known rho-pi-axial coupling constant (a needed input to the calculation) in order to give the desired residue. Such a change affects the predictions in all kinematic regions, and therefore the Argonne results did indeed provide discrimination between the two models. It was found that Model $B$ (the new model with PCAC incorporated by the choice of coupling constant) agrees significantly better than Model $A$ (the previous model not entirely consistent with PCAC) with the Argonne data. Thus, indirect support is provided for PCAC.

In addition to the work described above, effort was also directed to the area of gauge field theories. An attempt was made to follow the recent developments and become acquainted with the calculational techniques. The goal of this inquiry is to do a calculation of weak production combining the successful dynamical model above with the gauge field theories of the lepton interactions, obtaining detailed predictions (especially for neutral current reactions such as $\left.v+p \rightarrow v+p+\pi^{0}\right)$ to be compared with experiment. This effort is continuing at the present time. References

1. P. A. Zucker, Phys. Rev. D4, 3350 (1971).

2. I. Budagov et.al, Phys. Letters 29B, 524 (1969):

3. J. Campbell et. al., Phys. Rev. Letters 30, 335 (1973).

4. P. Schreiner and F. von Hippel, Phys. Rev. Letters 30, 339 (1973) and Nuçear Physics B58, 333 (1973). 
H. Courant, J. Kintner, Y. Mlakdisi, E. Plarquit, M. Marshak, E. Peterson, K. Ruddick

The experimental group has been involved primarily in the operation of E-305 at ARgonne National Laboratory. This was an experiment to study $\phi$ meson production in the interaction between negative pions and protons at 9 different incident pion momenta, from threshold at $1.6 \mathrm{GeV} / \mathrm{c}$ to a maximum of $3.5 \mathrm{GeV} / \mathrm{c}$.

This process is forbidden by a simple quark model and, indeed, it is known that at high incident momenta $(\geq 5 \mathrm{GeV} / \mathrm{c})$ the production cross-section is 2-3 orders of magnitude lower than the comparable $\omega$-meson production. Sparse bubble chamber data indicate a much smaller difference near threshold. It was the aim of the experiment to elucidate the nature of production in the region. below $3.5 \mathrm{GeV} / \mathrm{c}$, where the cross-section must change very rapidly.

The detection system for E-305 consisted of a double-arm magnetic spectrometer with large volume gas Cerenkov counters to identify the $\mathrm{K}^{+} \mathrm{K}^{-}$decay mode of the $\phi$ meson. Information on the trajectories of all charged particles involved in the interaction was taken from multiwire proportional chambers (approximately 2000 wires) and read directly into our PDP-9 computer. Instantaneous monitoring of the operation of the experiment was an essential feature.

This was the first on-line experiment undertaken by our group. This, combined with the complexity of the experiment led to a number of unforeseen problems which were eventually overcome. Data-taking began early in 1973, and was completed in September, 1973. Despite the fact that data runs were of short duration and that the incident beam intensity was less than half than had been scheduled, due to accelerator problems, it is anticipated that the data will 
comprise 100-500 $\phi$ mesons at each of the 9 momenta. Approximately 500 data tapes were obtained, each tape containing several thousand triggers.

In addition to $\phi$ meson production, the data tapes will include approximately $10,000 \pi^{-} \mathrm{p} \rightarrow \pi^{+} \pi^{-} n$ events at low momentum transfer and low $\pi^{+} \pi^{-}$mass, which will provide information on the $\pi^{+} \pi^{-}$phase shifts near threshold. This data was taken at $3.5 \mathrm{GeV} / \mathrm{C}$ incident momentum where $N^{*}$ production does not interfere with a study of the $\pi^{+} \pi^{-}$system.

We also have data on the reactions $K^{-} p \rightarrow \phi \Lambda, \pi^{-} p \rightarrow K^{0} \Lambda, \Sigma$ and $\pi^{-} p \rightarrow \Delta^{0} \pi^{0}$. In this latter reaction, the $p \pi^{-}$decay of the $\Delta^{0}$ was detected in the spectrometer, with the $\Delta^{0}$ produced at small forward angles in the lab, corresponding to large momentum transfer, and will provide information on a baryon exchange process not previously studied in any detail.

The recent acqusition of a magnetic tape unit will make it possible to transfer data from our DEC tapes to a form compatible with the University of Minnesota CDC 6600 computer which will be used for the final data analysis. Anaiysis programs will be tested on the PDP-9 before large-scale data processing begins.

Data analysis will take several months.

Some work has continued on analysis of data from an optical spark chamber experiment E-219, which we completed several years ago at Argonne. Final analysis of the interactions leading to $\pi^{-} p+\pi^{0} \gamma_{n}$ and $n \gamma_{n}$ is complete. The results are essentially the same as we reported at the NAL conference in September 1972. These provide considerably improved upper limits for the electromagnetic decays of $\omega$ and $\rho$ mesons to $n$ meson plus gamma ray. We are also searching for the decay $n \rightarrow \pi^{0} \gamma r$, and as a preliminary result have found that 
this decay is certainly much less probable than previously measured. Our experiment was the first to provide an overconstrained fit to this rare decay mode of the $n$ meson.

The data from experiments E-219 and E-305 will provide the basis for Ph.D. theses by J. Kintner and Y. Makdisi.

Instrumentation development has been minimal during the operation of E-305. Some work has been carried out to find an inexpensive means for analog to digital conversion of pulse heights suitable for transfer to the computer. Such devices will be required for certain future experiments that we are considering, including our proposed experiment at the National Accelerator Laboratory.

$K^{\circ} p$ Interactions - E. Coleman

This is the first experiment to look at $k^{\circ} p$ interactions in the resonance region. A total of 2.4 million pictures have been taken in collaboration with groups at Hamburg, Heidelberg, and Tel-Aviv. The neutral kaon beam with some ne neutron background was produced by a $4.5 \mathrm{GeV} / \mathrm{c}$ electron beam incident upon a beryllium target. The charged particles were bent from the beam and the gammas were eliminated by $31 \mathrm{~cm}$ of lead in the beam path. Eighty percent of the kaons had momenta between 0.5 and $2.5 \mathrm{GeV} / \mathrm{c}$ with a peak at $1.5 \mathrm{GeV} / \mathrm{c}$. nuring the past

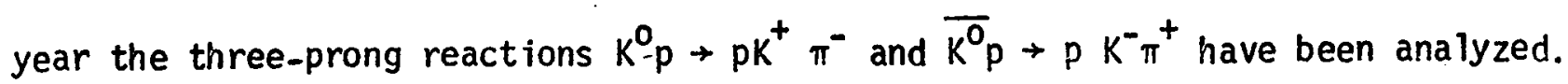
These $k^{*}$ production can be described by $\pi$ and $\omega$ exchange contributions because the $\rho$ and $\phi$ contributions are relatively small. The $\Delta$ production can be described by $\rho$ exchange. The analys is of heavier resonances is underway. 
The $\mathrm{K}^{0} \mathrm{p} \rightarrow \mathrm{p} \mathrm{K}^{+} \pi^{-}$mass plots showed strong signals due to $\mathrm{K}^{*_{0}}(890)$ for $\mathrm{K}^{+} \pi^{-}$ and due to $\Delta^{0}(1236)$ for $\mathrm{p}_{\pi}^{-}$. The $t$ distribution when fitted to the function

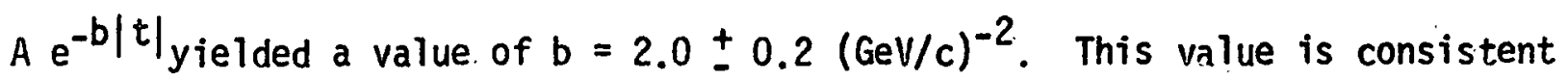
with predictions of the Regge model. Analysis of the angular correlations yielded the spin density matrix elements $\rho_{00}=0.78 \pm 0.08$ and $\rho_{1,-1}=0.16 \pm 0.04$. The $\Delta^{0}$ channel was complicated by reflections from the $K^{* 0} p$ channel; however, its structure was studied via the Sakuri-Stodolsky model. The data in the region $1.18 \mathrm{GeV} / \mathrm{c}^{2}<\mathrm{m}\left(\mathrm{p \pi ^{- }}\right)<1.28 \mathrm{GeV} / \mathrm{c}^{2}$ did not agree with the Sakuri-Stodolsky model. The value of $\operatorname{Re} p_{3,-1}=0.10 \pm 0.05$ derived from the data was much smaller than the value 0.216 predicted by the model. The ratio $B / A$ from fitting the data to $A+B \cos ^{2} \theta$ using the Sakuri-Stodolsky coordinate system was found to be $0.71 \pm 0.07$ instead of 3 as predicted by the model. The reaction $\overline{K^{0}} p \rightarrow p K^{-} \pi^{+}$shows clear signals due to $\overline{K^{*}}(890), \Delta^{++}(1236)$, $\gamma^{*}(1520)$ and $\gamma^{*}(1820)$. Fitting the events in the region 840 to $960 \mathrm{GeV} / \mathrm{c}^{2}$, $\mathrm{K}^{* 0}$ region, to the function $\left.A \mathrm{e}^{-\mathrm{b}|t|}\right|_{\text {yielded the value } \mathrm{b}}=2.1 \pm 0.2(\mathrm{GeV} / \mathrm{c})^{-2}$. The decay angular correlations of $\mathrm{K}^{* 0}$ yielded the spin density matrix element $\rho_{i,-1}=0.16 \pm 0.09$. The decay angular correlations of $\Delta^{++}(1236)$ showed Re $\rho_{3,-1}=0.205 \pm 0.08$ which is consistent with the Sakuri-Stodolsky prediction of 0.216 .

np Interactions - E. Coleman

The reactions,$n p \rightarrow p p \pi^{-}$and $n p \rightarrow d \pi^{+} \pi^{-}$were studied as a by-product of the analys is of three-prong events in the kaon film. For the first time one is able to look at the decay angular distributions of vertex separated $\pi^{-p}$ systems free of interferences around $2.0 \mathrm{GeV} / \mathrm{c}(n \mathrm{p} \rightarrow \mathrm{pp} \pi)$ in this experiment. We assign 
the $\pi^{-}$pion once to the neutron vertex and then to the proton vertex and then we treat every event as contributing part of the time to the neutron vertex and part of the time to the proton vertex. This made it possible to make a sensitive search for off-shell I = 1/2 partial wave scattering in the virtual pion nucleon scattering processes $\pi^{0} n \rightarrow \pi^{-} p$ and $\pi^{-} p \rightarrow \pi^{-} p$. The decay distribution parameters of the $\pi^{-} p$ system as a function of four-momentum transfer were determined for both the neutron and proton vertices. Fitting the data to the function $A \cdot e^{-b \Delta^{2}}$ for $\Delta^{2}>0.01(\mathrm{GeV} / \mathrm{C})^{2}$ yielded $A=185$ and $b=5.5$ for the neutron vertex and $A=190$ with $b=5.6(\mathrm{GeV} / \mathrm{c})^{-2}$ for the proton vertex. The values 5.5 and $5.6(\mathrm{GeV} / \mathrm{C})^{-2}$ are typical of pion exchange process which generally have $b$ values between 4 and $6(\mathrm{GeV} / \mathrm{c})^{-2}$. The $n p \rightarrow d \pi^{+} \pi^{-}$data indicated a possible $d \pi$ resonance of mass $2.14 \mathrm{GeV} / \mathrm{c}^{2}$ and width $54 \mathrm{MeV}$; from SU(6) Dyson and Xuong expect such a resonance in their classification scheme. A fit of the $\mathrm{d} \pi^{+} \pi^{-}$data to $A+B \cos ^{2} \theta$ yielded $A=0.84$ and $B=0.70$. The $\pi^{+} \pi^{-}$. mass distributions indicated that the $\pi^{+}$and $\pi^{-}$originate from two different vertices. A Ph.D. thesis (T. Fesesse, June 1973) has been written on the threeprong kaon and neutron interactions.

Momentum Spectrum for $5.53 \mathrm{GeV} / \mathrm{C}$ Inelastic Pion-Deuteron Collisions - E. Coleman

The momentum spectrum for pion deuteron collisions was measured at the zerogradient synchrotron of Argonne National Laboratory for $5.53 \mathrm{GeV} / \mathrm{C}$ incident pions. The beam intensity was $2 \times 10^{5}$ pions/pulse repeated at the rate of 1000 pulses $/ \mathrm{h}$. A single-arm spectrometer detected the scattered pions, and a veto counter downstream from the target eliminated pions which did not interact with the target. The events satisfying the criteria for a good event were stored in a multichannel 
analyzer. The scattering of pions in an empty target and in a carbon target was used to determine the background. Corrections to the data were applied for scattered pion decay, attenuation of the pion beam, muon and electron contamination of the beam, nuclear absorption of the scattered pion, impurities in the liquid-deuterium target, and electronic deadtime due to the veto counter. The results of this experiment were compared with the predictions of various eikonal models. Unlike the elastic scattering, double scattering contributions are very small away from the forward direction. The best theoretical results were obtained with the Glauber high energy approximation. These results will be published soon in Physical Review.

Hadron-Deuteron Collisions and Deuteron Structure - E. Coleman

The contributions of the single and double scattering components of elastic scattering of hadrons by deuterons depends very strongly on the structure of the deuteron wave function. For four-momentum transfers between 0.2 and $0.7(\mathrm{GeV} / \mathrm{c})^{2}$, the magnitude of the D-state in the wave function can change the differential cross section by almost an order of magnitude. The sensitivity of this region is being exploited to determine the percentage of D-state in the total deuteron wave function when only S- and D-state contribute. Such effects as variation of the hadron-nucleon scattering amplitude with scattering angle, off-mass-shell effects, Fermi motion, and final state interactions are still being studied. The effects of relativistic deuteron wave functions and non-local potentials are also being investigated. A review article sumarizing these findings is being prepared. 


\section{PUBLICATIONS}

C00-1764- 167 "Non-local and Majorana-Exchange Terms in the Optical Mode1", Y. Tang and D. Thompson, Phys. Rev. C6, 2057 (1972).

C00-1764-178 "Phenomenological Study of S-Shell Hypernuclei with $N$ and $N N$ Potentials", Y. Tang and D. Thompson, Nucl. Phys. B47, 109 (1972). (Previous ly report last year under C00-1764-168 by mistake)

C00-1764-179 "Formulation of Bethe-Salpeter Equation in e-p neep Inelastic Scattering", W. Lau. (submitted to Nuovo Cimento)

C00-1764-180 "Microscopic Study of the Five - Nucleon Systems", F. Chwieroth.

C00-1764-181 "Derivation of General Conservation Laws and Ward-Takahashi Identities in the Functional Integration Method", H. Suura and B. L. Young.

CO0-1764-182 "Odd-Even Absorption in ${ }^{3} \mathrm{He}+{ }^{3} \mathrm{He}$ Scattering and Level Structure of ${ }^{6} \mathrm{Be}^{\prime}$, Nucl. Phys. A201, 301 (1973), Y. Tang and D. Thompson.

C00-1764-183 "Study of $d+{ }^{3} H$ and $d+{ }^{3}$ He Systems with the Resonating-Group llethod", Y. Tang and D. Thompson, Phys. Rev. 으, 938 (1973). C00-1764-184 "Distortion Effects in $d+$ System", will appear in November issue of Phys. Rev. C., Y. Tang.

c00-1764-185 "Effects of Imaginary Potentials on Spurious Resonances in Resonating-Group Calculations", will appear in Phys. Letter, Y. C. Tang.

C00-1764-186 Microscopic Coupled-Channel Study of the Five-Nucleon Sys tem with the Resonating-Group Method", will appear in Phys. Rev., Y. C. Tang.

C00-1764-187 Progress Report. 
C00-1764-188 "Regge Trajectories and the Quark-Gluon Coupling Constant", $D$. Coon and H. Suura.

C00-1764-189 "Short-distance behaviour of Quantum Electrodynamics and the Callan-Symanzik Equation for the Photon Propagator", by Rafael and Rosner.

C00-1764-190 "Further Evidence for a 70, L = 2 Baryon Multiplet", by Rosner and Weyers.

C00-1764-191 "Question of Direct Measurement of the Quark Charge", by G. Farrar 8. J. Rosner, Phys. Rev. D7 (1973) 2747.

C00-1764-192 "Resonances in High Energy Colliding Lepton Beams", J. Rosner: CO0-1764-193 "Asymptotic Behavior of Trajectory Functions", Chang and Rosner. CO0-1764-194 "Higher Symmetries and Baryon Resonances", J. Rosner. 